\title{
The Importance of Flavonoids in Ruminant Nutrition
}

\author{
Kalantar $\mathbf{M}^{1,2 *}$ \\ ${ }^{1}$ Department of Animal Science, Qom Agricultural and Natural Source research and Education Center, Qom, Iran \\ ${ }^{2}$ Department of Animal Science, University of Applied Science and Technology, Qom, Iran
}

*Corresponding author: Kalantar M, Department of Animal Science, Qom Agricultural and Natural Source research and Education Center (AREEO), Qom, Iran. P.O. Box 195. Tel: +982536618220 .

Received Date: October 08, 2018

Published Date: November 26, 2018

\begin{abstract}
Recent scientific reports indicated that flavonoid could affect the rumen volatile fatty acids production along with a reduction in rumen methane concentration which resulted in improvement of performance and production. Flavonoids as polyphenolic compounds are powerful antimicrobials and antioxidants with anti-inflammatory and immune system benefits. Flavonoids can also promote the growth and development of animals as well as improve the quality of animal products. Therefore, these compounds are widely used as feed additives in animal production instead of antibiotics. Base on the research findings, addition of the flavonoids to ruminate diets could suppress methane production without influencing rumen microbial fermentation, fatty acid production and performance of beef or dairy cattle. Flavonoids are capable of the improvement of volatile fatty acids production together with a reduction in both rumen ammonia concentration and methane production which are considered as desirable changes in rumen environment. Also, the positive effect of flavonoids on rumen microbial fermentation and nutritional stress such as bloat or acidosis have been well demonstrated. Many factors such as chemical structure, absorption, its distribution and elimination determine the bioavailability of flavonoids. Isoflavones and flavanols seems to have lower bioavailability, whereas higher bioavailability of proanthocyanidins in ruminants were reported. Incorporation of flavonoids into dairy/beef products may provide a novel way to increase quantity and quality of future products of dairy farming.
\end{abstract}

\section{Introduction}

Flavonoids as benzo-pyrone derivatives of phenolic compound are included of alarge family of thousands hydroxylated polyphenolic compounds. They have the general structure of a 15-carbon skeleton, which consists of two phenyl rings and heterocyclic ring [1]. The majority of flavonoids generally remain conjugated with sugars as glycosides [2,3]. Flavonoids have different roles in plants that includes help at pollination, reduce environmental stress, disposal of microbial infection and regulating cell growth $[1,4,5]$. Flavonoids are an important class of phytochemicals products found in most of herbs, fruits, vegetables and certain beverages. Certain plant-based food groups are known to be much higher in flavonoids family than others including some fruits (berries and apples, tree fruits, citrus fruits, tropical fruits), vegetables (all type of green vegetables, especially brussels cabbage- type sprouts and spinach), spices, nuts and beans, grains (cereals, soy and beans), beverages, tea of all types, acid forming foods, garlic and onion $[1,2,3,4,5]$.
Some of the well-known flavonoids are quercetin, kaempferol and anthocyanidins. They can be classified into three main classes including bioflavonoids, isoflavonoids, and neoflavonoids [4,6,7]. Flavonoids are considered as promoter of the growth and enhancer of the products quality of animal due to its anti-microbial and antioxidative properties $[8,9]$. There is substantial interest to study the potential of natural bioactive compounds to modify ruminal microbial ecosystem and desirable changes in the fermentation conditions such as $\mathrm{pH}$, propionate concentration, and protein degradation [10-13]. Recently, the flavonoids and the other members of phenolic compounds are widely used as feed additives in ruminant production $[5,14,15,16]$.

\section{Metabolism and bioavailability of flavonoids in ruminants}

Flavonoids are transported to the liver after absorption, where they form glucuronide, sulfate or methyl-conjugates compounds 
before being excreted through the urine or faeces $[8,9,17,18]$. This is well illustrated that the catechin and epicatechin as monomeric components of flavanols are the only derivatives absorbed in monogastrics $[7,19]$. In contrast to monogastrics, it was shown that ruminants can benefit from the strong antioxidant properties of polymeric proanthocyanidins by metabolizing them into bioavailable compounds with epicatechin as intact flavonoid-ring structure [17,18,20,21,22].

Several factors such as chemical structure, absorption rate, the level of distribution and elimination determine the biological effects of flavonoids $[7,17,23]$. The higher bioavailability of isoflavones and flavonols has been observed in monogastric compared with the other subclasses of flavonoids, while anthocyanins are reported to have the less bioavailability $[4,24]$. whereas, it was described higher proanthocyanidins bioavailability in ruminants compared to other flavonoids subclasses [4,14,16,22].

\section{Significance of Flavonoids in Ruminant Ration}

Recent advances in ruminant nutrition researches suggest that to control metabolic processes in the rumen to make it more efficient using natural feed additives such as flavonoids is desirable $[14,15,16]$. The improvement of volatile fatty acids production along with a decrease in both rumen ammonia and methane concentration are considered as desirable changes in rumen ecosystem $[13,25,26]$.

The positive effect of flavonoids and phenolic compounds (saponins, tannins, essential oils, organosulphur containing compounds) on the productivity and health of animals as well as rumen fermentation and control of nutritional stress such as bloat and acidosis have been demonstrated in several studies $[27,28]$. Flavonoids as polyphenolic compounds have similar pattern of action similar to monensin and other type of antibiotics $[29,30,31,32]$. Higher rumen $\mathrm{pH}$ values were observed in high grain heifers diets supplemented with flavonoids compared to the control group, which likely due to the beneficial effect of flavonoid in enhancing lactate-consuming microorganisms i.e. M. elsdenii $[12,14]$.

\section{Effect on milk production}

Both rumen microbial ecosystem and ruminant's performance can be influenced by the use of flavonoids in the diets. Generally, flavonoids can modify the fermentation characteristics [10,12,33], and can be served as anti-microbial agent to decrease pathogens and methane producing bacteria as well as improve the antioxidant content to decrease lipid oxidation and increase the quality of the milk/meat production $[13,14,26]$.

Incorporation of moderate levels of flavonoids/polyphenols into milk/meat and dairy products through the feeding of animals with food sources rich in flavonoids and phytochemicals, may provide a novel way to increase flavonoids/polyphenol content of daily food for consumers $[7,14]$. It was reported that the increase in milk yield and lactation performance in dairy cows were observed after administration of sylimarin in the feed $(10 \mathrm{~g} / \mathrm{d})$ which mainly consist of flavonolignans [34]. It is well reported that flavonoids can enter the circulation, where they may be spread across tissues and finally be renally excreted [35]. The other fate of absorbed flavonoids is the mammary gland, where they can incorporated into the milk of lactating animals [17,23,34]. Previous studies have demonstrated that the positive effect of various food processing waste and plant origin byproducts on the production and composition of milk $[14,16,26]$.

\section{Control of rumen production}

The commonly used feed additives in ruminant nutrition have an important role as modulators of the end products of rumen fermentation $[11,13,30]$.

Some rumen fermentation dysfunctions such as acidosis or bloat can cause after consumption of high-concentrate diets [16]. The dietary inclusion of monensin/antibiotics appears to reduce the incidence of rumen dysfunctions. However, use of antibiotics as feed additives were banned by the European Community since January 2006 [14]. From that time many feed additives such as flavonoids have been proposed as alternatives to antibiotic therapies [12,14,16,25,30,31].

In association with the anti-microbial properties of flavonoids extracts, the use of flavonoids extracts to rumen fermentation have been the subject of many experiments $[10,16,6,30]$. Using mixtures of plants flavonoids in continuous rumen culture system can modify fermentation conditions including $\mathrm{pH}$, propionate proportion and/or protein degradation, although the results were not always homogeneous $[11,12,13,14]$.

\section{Diminution of methane production}

A large population of methanogenic bacteria present in the rumen have been implicated in global warming, and high attempts to moderate rumen microbial fermentation towards reducing the methane production through application of feed additives remain a high priority $[14,25,26]$. Decrease in methane production have been considered as a valid index of rumen microbial fermentation inefficiency $[13,14]$. Furthermore, changes in VFA profile, i.e. improvement in the molar proportion of propionate to the expense of acetate, will be coming to reduction in $\mathrm{CH}_{4}$ synthesis $[26,28]$. Moreover, $\mathrm{CH}_{4}$ is a potent greenhouse gas with global warming capability and is 25 times more toxic than $\mathrm{CO}_{2}[25,14,16]$.

Previous reports evidenced that the addition of plants extracts rich in secondary compounds such as saponins, tannins, essential oils and also extracts rich in flavonoids reduced rumen $\mathrm{CH}_{4}$ production $[25,14,15]$, showed that the addition of the flavonoids naringin and quercetin to ruminate diets could suppress methane production without influencing rumen microbial fermentation [14]. However, uncertainties in such processes arises from primarily, mechanism of methane depression are unidentified and secondarily, the plant extracts are constituted by complex mixtures whose action on rumen fermentation may derive from the synergistic and/or antagonistic action of the flavonoids/polyphenol mixtures $[10,11]$. Currently, various flavonoids/polyphenol rich feed additives 
suppressing methane producing are available in the market However, these products mainly contain plant crude extracts, and it is rather difficult to determine the response of rumen microbes to the flavonoids/polyphenols. The presence of other components such as glycosides, phenolics, terpenoids, alkaloids, essential oils, and organic acids in the plant extracts may affect the results. In addition, awareness of the effect of flavonoids in the pure form on rumen microbial activity is still incomplete $[12,13,14,26]$.

\section{Conclusion}

Flavonoids due to its anti-microbial and anti-oxidative properties are widely used as feed additives in animal production. They have potential to modify rumen microbial activity and desirable changes in the fermentation conditions such as $\mathrm{pH}$, propionate proportion, and protein degradation. The positive effect of flavonoids and phenolic compounds on the productivity and health of animals as well as rumen fermentation, reduce methane production, and control of nutritional stress such as bloat and acidosis have been demonstrated in several studies. According to the recent findings, incorporation of flavonoids/polyphenols into milk/meat and dairy products using food sources rich in flavonoids and phytochemicals, may provide a novel way to increase flavonoids/polyphenol consumption and consequently promote health of society, particularly for people with a low diet of flavonoids/polyphenol content.

\section{Acknowledgment}

None.

\section{Conflict of Interest}

No Conflict of Interest.

\section{References}

1. Xiao J, Kai G, Yamamoto K, Chen X (2013) Advance in dietary polyphe 7 nols as $\alpha$-glucosidases inhibitors: a review on structure-activity relationship aspect. Crit Rev Food Sci Nutr, 53(8): 818-836.

2. Manach C, Scalbert A, Morand C, Remesy C, Jimenez L (2004) Polyphenols: food sources and bioavailability. Americam Journal of Clinical Nutrition 79(5): 727-747.

3. Manach C, Williamson G, Morand C, Scalbert A, Remesy C (2005) Bioavailability and bioefficacy of polyphenols in humans. I. Review of 97 bioavailability studies. Americam Journal of Clinical Nutrition 81(1 Suppl): 230S-242S.

4. Kumar S, Pandey AK (2013) Chemistry and biological activities of flavonoids: an overview. Scientific World Journal p:16.

5. Zhan J, Liu M, Su X, Zhan K, Zhang C, Zhao G (2017) Effects of alfalfa flavonoids on the production performance, immune system, and ruminal fermentation of dairy cows. Asian-Australas J Anim Sci, 30(10): 14161424.

6. Panche AN, Diwan AD, Chandra SR (2016) Flavonoids: an overview. Journal of Nutritional Science 5: e47.

7. Kalantar M, Ahmadipour B, Kalantar MH (2018) Flavonoids as a potent anti-oxidant in Nutrition: A Mini-Review. Appro Poult Dairy \& Vet Sci 4(1):1-3.

8. Ahmadipour B, Hassanpour H, Asadi E, Khajali F, Rafiei F et al. (2015) Kelussia odoratissima Mozzaf- A promising medicinal herb to prevent pulmonary hypertension in broiler chickens reared at high altitude. J Ethnopharmacol 159:49-54.
9. Ahmadipour B, Kalantar M, Hosseini SM, Yang LG, Kalantar MH, et al. (2017) Hawthorn (Crataegus Oxyacantha) Extract in the Drinking Water of Broilers on Growth and Incidence of Pulmonary Hypertension Syndrome (PHS). Brazil J Poult Sci 19(4):639-644.

10. Broudiscou LP, Papon Y, Broudiscou AF (2002) Effects of dry plant extracts on feed degradation and the production of rumen microbial biomass in a dual outflow fermenter. Animal Feed Science and Technology 101(1-4): 183-189.

11. Busquet M, Calsamiglia S, Ferret A, Kamel C (2006) Plant extracts affect in vitro rumen microbial fermentation. J Dairy Sci 89(2): 761-771.

12. Balcells J, Aris A, Serrano A, Seradj AR, Crespo J, et al. (2012) Effects of an extract of plant flavonoids (Bioflavex) on rumen fermentation and performance in heifers fed high-concentrate diets. Journal of Animal Science 90(13): 4975-4984.

13. Oskoueian E, Norhani A, Oskoueian A (2013) Effects of flavonoids on rumen fermentation activity, methane production, and microbial population. BioMed Research International 2013: p: 8

14. Seradj AR, Abecia L, Crespo J, Villalba D, Fondevila M, Balcells J (2014) The effect of Bioflavex® and its pure flavonoid components on in vitro fermentation parameters and methane production in rumen fluid from steers given high concentrate diets. Anim Feed Sci Technol 197: 85-91.

15. Paula EM, Samensari RB, Machado E, Pereira LM, Maia FJ (2016) Effects of phenolic compounds on ruminal protozoa population, ruminal fermentation, and digestion in water buffaloes. Livest Sci 185:136-41.

16. Lee SHY, Humphries DJ, Cockman DA, Givens DI, Spencer JPE (2017) Accumulation of citrus flavanones in bovine milk following citrus pulp incorporation into the diet of dairy cows. EC Nutrition 7(4): 143-154.

17. Gonzales GB, Smagghe G, Grootaert C, Zotti M, Raes K, et al. (2015) Fla-vonoid interactions during digestion, absorption, distribution and me-tabolism: a sequential structure-activity/property relationshipbased approach in the study of bioavailability and bioactivity. Drug Metab Rev 47(2): 175-190.

18. Spencer JPE, Abd El Mohsen MM, Minihane AM, Mathers JC (2008) Biomarkers of the intake of dietary polyphenols: Strengths, limitations and application in nutrition research. British Journal of Nutrition 99(1): $12-22$.

19. Nakamura Y, Tonogai Y (2003) Metabolism of Grape Seed Polyphenol in the Rat. Journal of Agricultural and Food Chemistry 51(24): 7215-7225.

20. Gladine C, Morand C, Rock E, Bauchart D, Durand D (2007) Plant extracts rich in polyphenols (PERP) are efficient antioxidants to prevent lipoperoxidation in plasma lipids from animals fed $n-3$ PUFA supplemented diets. Animal Feed Science and Technology 136(3-4): 281-296.

21. Gladine C, Morand C, Rock E, Gruffat D, Bauchart D (2007) The antioxidative effect of plant extracts rich in polyphenols differs between liver and muscletissues in rats fed n-3 PUFA rich diets. Animal Feed Science and Technology 139(3-4): 257-272.

22. Gladine, C, Rock E, Morand C, Bauchart D, Durand D (2007) Bioavailability and antioxidant capacity of plant extracts rich in polyphenols, given as a single acute dose, in sheep made highly susceptible to lipoperoxidation. Brit J Nutr 98(4): 691-701.

23. Heim KE, Tagliaferro AR, Bobilya DJ (2002) Flavonoid antioxidants: Chemistry, metabolism and structure-activity relationships. Journal of Nutritional Biochemistry 13(10): 572-584.

24. Hollman PCH (2004) Absorption, bioavailability, and metabolism of flavonoids. Pharmaceutical Biology 42: 74-83.

25. Patra AK, Saxena J (2010) A new perspective on the use of plant secondary metabolites to inhibit methanogenesis in the rumen. Phytochemistry, 71(11-12): 1198-1222

26. Bodas R, Prieto N, Garcia-Gonzalez R, Andres S, Giraldez FJ, et al. (2012). Manipulation of rumen fermentation and methane production with plant secondary metabolites. Animal Feed Science and Technology 176(1-4):78-93. 
27. Rochfort S, Parker AJ, Dunshea FR (2008) Plant bioactives for ruminant health and productivity. Phytochemistry 69(2):299-322.

28. Patra AK, Kamra DN, Agarwal N (2006) Effect of plant extracts on in vitro methanogenesis, enzyme activities and fermentation of feed in rumen liquor of buffalo. Animal Feed Science and Technology, 128 (3-4): 276291.

29. Cushnie TPT, Lamb AJ (2005) Antimicrobial activity of flavonoids. International Journal of Antimicrobial Agents 26(5): 343-356.

30. Cushnie TPT, Lamb AJ (2011). Recent advances in understanding the antibacterial properties of flavonoids. Int. J. Antimicrob. Agents 38(2): 99-107.

31. Yaghoubi SMJ, Ghorbani GR, Rahmani HR, Nikkhah A (2007) In vitro manipulation of rumen fermentation by propolis flavonoids and monensin. J Dairy Sci 90(1): 105-106.
32. Yaghoubi SMJ, Ghorbani GR, Rahmani HR, Nikkhah A (2008) Growth, weaning performance and blood indicators of humoral immunity in Holstein calves fed supplemental flavonoids. J Anim Physiol Anim Nutr, 92 (4): 456-462.

33. Ahn J, Grün IU, Fernando LN, (2002) Antioxidant properties of natural plant extracts containing polyphenolic compounds in cooked ground beef. Journal of Food Science 67(4): 1364-1369.

34. Tedesco D, Tava A, Galletti S, Tameni M, Varisco G et al. (2004) Effects of silymarin, a natural hepatoprotector, in periparturient dairy cows. Journal of Dairy Science 87(7): 2239-2247.

35. Martin AK (1982) The origin of urinary aromatic compounds excreted by ruminants. 2 . The metabolism of phenolic cinnamic acids to benzoic acid. British Journal of Nutrition 47(1): 155-164. 